\title{
Strain-Promoted 1,3-Dipolar Cycloaddition of Cycloalkynes and Organic Azides
}

\author{
Jan Dommerholt ${ }^{1} \cdot$ Floris P. J. T. Rutjes ${ }^{1}$. \\ Floris L. van Delft ${ }^{2}$
}

Received: 24 November 2015/ Accepted: 17 February 2016/Published online: 22 March 2016

(C) The Author(s) 2016. This article is published with open access at Springerlink.com

\begin{abstract}
A nearly forgotten reaction discovered more than 60 years ago- the cycloaddition of a cyclic alkyne and an organic azide, leading to an aromatic triazole-enjoys a remarkable popularity. Originally discovered out of pure chemical curiosity, and dusted off early this century as an efficient and clean bioconjugation tool, the usefulness of cyclooctyne-azide cycloaddition is now adopted in a wide range of fields of chemical science and beyond. Its ease of operation, broad solvent compatibility, $100 \%$ atom efficiency, and the high stability of the resulting triazole product, just to name a few aspects, have catapulted this so-called strain-promoted azide-alkyne cycloaddition (SPAAC) right into the top-shelf of the toolbox of chemical biologists, material scientists, biotechnologists, medicinal chemists, and more. In this chapter, a brief historic overview of cycloalkynes is provided first, along with the main synthetic strategies to prepare cycloalkynes and their chemical reactivities. Core aspects of the strain-promoted reaction of cycloalkynes with azides are covered, as well as tools to achieve further reaction acceleration by means of modulation of cycloalkyne structure, nature of azide, and choice of solvent.
\end{abstract}

Keywords Strain-promoted cycloaddition $\cdot$ Cyclooctyne $\cdot$ BCN $\cdot$ DIBAC $\cdot$ Azide

This article is part of the Topical Collection "Cycloadditions in Bioorthogonal Chemistry"; edited by Milan Vrabel, Thomas Carell

Floris P. J. T. Rutjes

f.rutjes@science.ru.nl

$\bowtie$ Floris L. van Delft

floris.vandelft@wur.nl

1 Radboud University, Nijmegen, The Netherlands

2 Wageningen University and Research Centre, Wageningen, The Netherlands 


\section{Introduction}

The spontaneous reaction of cycloalkynes with an organic azide, in all its simplicity, is a fascinating organic chemical transformation. Simply by mixing and stirring, without the necessity of reagents, catalysts, or carefully controlled reaction conditions, a stable triazole product is formed by fast and selective cycloaddition of cycloalkyne with azide. As will become clear throughout this chapter, the latter reaction has now firmly established itself as a powerful and versatile chemical process with broad academic and commercial applications. Core to the chemistry lies a highly strained, medium-sized cyclic alkyne, most prominently a cyclooctyne. In this chapter, the synthesis and chemical reactivity of cyclic alkynes is broadly delineated, with particular emphasis on undoubtedly the most important of applications of cycloalkynes: cycloaddition with an organic azide, leading to the formation of a stable triazole.

\section{The Fascinating Chemistry of Cycloalkynes}

\subsection{Conception of Cycloalkynes}

In the second half of the previous century, interest emerged at several laboratories around the world to explore the synthesis and properties of medium-sized cycloalkynes. Pioneers in the field, Blomquist et al., at Cornell University (USA), convincingly demonstrated in 1951 that plain cyclononyne and cyclodecyne could be accessed by oxidative decomposition of the respective cycloalka-1,2-diones, and isolated in pure form by distillation [7]. Two years later, the same group also reported the successful preparation of the eight-membered ring acetylene [8], while similar explorations on cycloalkynes were reported by Prelog and colleagues at the ETH in Zurich (Switzerland) around the same time [44]. It must be noted that the preparation and isolation of cyclooctyne had been claimed by Domnin (USSR) some 15 years earlier [21], but the reported characterization data suggest that the compound was - at bestobtained as a mixture with the isomeric 1,2-cyclooctadiene and other unsaturated hydrocarbons. In 1961, it was Wittig at the University of Heidelberg (Germany) who was the first to demonstrate the formation of the yet-smaller five-, six-, and sevenmembered cycloalkynes, as well as 1,2-dehydrobenzene, better known as benzyne [62].

The successful preparation of cycloalkynes also opened up the possibility to explore their unique chemical reactivity. In fact, the transient existence of the cycloalkyne species could initially only be indirectly corroborated by fast in situ trapping of the smaller-sized rings (seven carbons and below) before decomposition [31]. While not strictly applicable to cyclooctyne, which is the smallest cyclic alkyne that can be isolated and stored in pure form, Blomquist already noted that nevertheless careful exclusion of air was requisite to avoid rapid decomposition. More importantly, he was also the first to observe that "cyclooctyne reacts explosively when treated with phenyl azide, forming a viscous liquid product" [8]. This remark is in fact the first historic administration of a process that has now become known as strain-promoted azide-alkyne cycloaddition (SPAAC). 


\subsection{Synthetic Preparation of Cycloalkynes}

A range of different synthetic procedures is known to obtain medium-sized cycloalkynes, as covered by several reviews [38]; Tochtermann [25]. In this paragraph, only the most relevant procedures will be covered.

The first synthetic reports on cycloalkynes involved a base-catalyzed oxidative decomposition of bis-hydrazones, readily prepared from the respective precursor 1,2-cycloalkadiones by condensation with hydrazine (Fig. 1, top). As oxidant, mercury oxide is most typically applied, however $\mathrm{Ag}_{2} \mathrm{O}$ or $\mathrm{Pb}(\mathrm{OAc})_{4}$ are also suitable. An analogous procedure (Fig. 1, bottom) employs the tosylate (Ts) derivative of hydrazine, which upon condensation with the diketone under reflux conditions forms the tosylated aminotriazole intermediate. Acidic removal of the tosyl group, followed by lead-mediated oxidation, also forms the desired cycloalkyne ring.

A direct procedure to obtain a cycloalkyne from a cycloalkanone involves the conversion into semicarbazone, followed by oxidation with selenium dioxide. The resulting intermediate 1,2,3-selenadiazole can be isolated in pure form and will eliminate, upon heating to $170-220{ }^{\circ} \mathrm{C}$ (or refluxing ethylene glycol), elemental selenium and nitrogen, with formation of the cycloalkyne.

The most often applied and most reliable procedure to obtain a cycloalkyne (Fig. 2, bottom), as originally developed by Brandsma in the Netherlands, [11], involves the stepwise double dehydrohalogenation of a vicinal dihalogenide (typically bromide), which can be readily obtained from a cyclic alkene upon treatment with elemental halogen. In this case, a first E2-elimination may take place under mildly basic conditions, forming an intermediate alkene, which in turn will undergo a second elimination upon treatment with a strong base like sodium amide or potassium tert-butoxide. More conveniently even, in many cases both

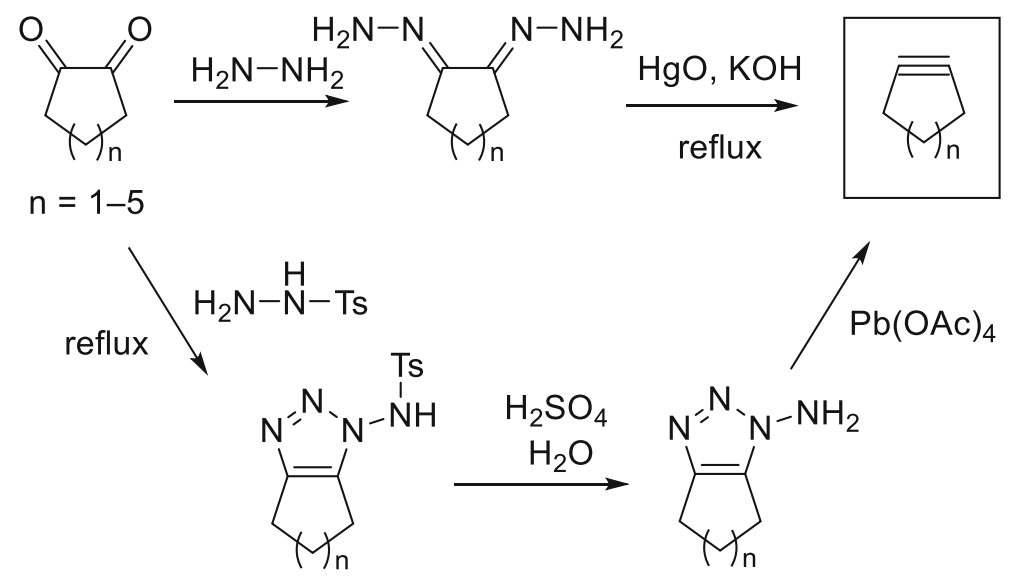

Fig. 1 Oxidative decomposition of cycloalka-1,2-dione dihydrazone, leading to medium-sized cycloalkynes $(n=1-5)$ 


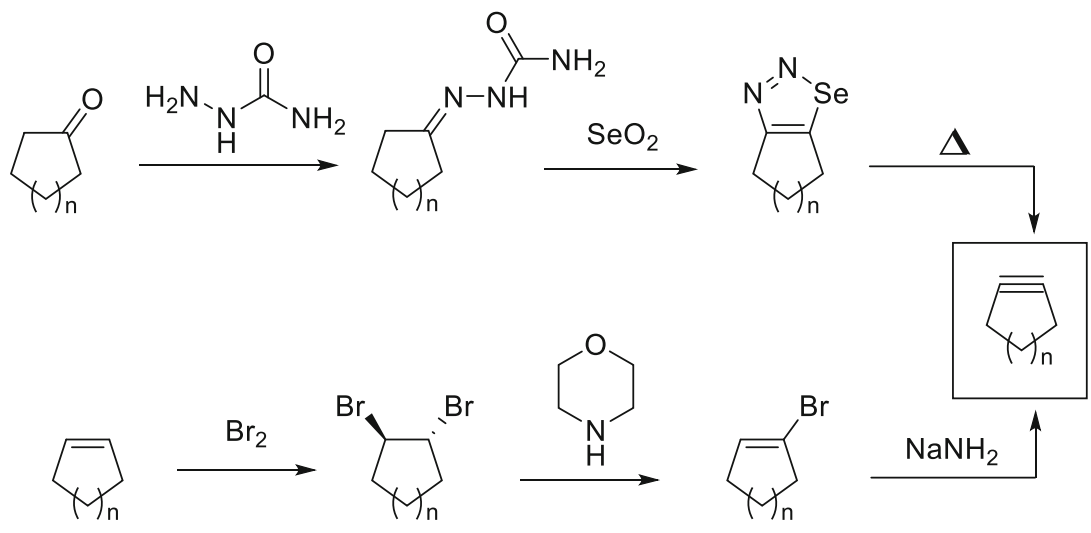

Fig. 2 Formation of cycloalkynes by fragmentation of 1,2,3-selenadiazole (top) or dehydrohalogenation (bottom)

eliminations can be induced in a one-step process upon treatment with a large excess of a strong base and/or heating.

Some more exotic synthetic processes for the synthesis of cycloalkynes, including reductive dehalogenation, photolytic elimination of bis-hydrazones or fragmentation of $\alpha, \beta$-epoxyketones have also been reported, but are beyond the scope of this chapter.

\subsection{Reactions of Cycloalkynes}

The stability of cyclic alkynes rapidly decreases with ring size. In fact, stability is directly correlated with the $\mathrm{C}-\mathrm{C} \mathrm{C}-\mathrm{C}$ bond angle, which, by virtue of the cyclic structure, cannot adopt the ideal $180^{\circ}$ bond angle of sp-hybridized carbon atoms. Interestingly, cyclooctyne was identified as the smallest isolable cycloalkyne, although its acetylene bond angle of $163^{\circ}$ still significantly deviates from linear. The experimentally determined ring strain of cyclooctyne is $\sim 18 \mathrm{kcal} / \mathrm{mol}$ [58], compared to $12.1 \mathrm{kcal} / \mathrm{mol}$ for saturated cyclooctane [6]. Not surprisingly, ringstrain is accountable for the intrinsically low stability of medium-sized cyclic alkynes, with calculated ring strains of $25 \mathrm{kcal} / \mathrm{mol}$ and above [6], leading to fast degradation and/or polymerization of seven-membered and smaller cycloalkynes, and thwarting their isolation in pure form. Gratifyingly, the same ring strain also imparts a unique reactivity profile onto medium-sized cycloalkynes, which may be advantageously employed in many ways as described here. In fact, the first synthetic preparation of seven-, six-, and five-membered cycloalkynes by Wittig et al. in [62] could only be corroborated by in situ generation of the formed alkyne in the presence of a suitable 'alkynophile' for fast $(4+2)$ cycloaddition [62]. In particular, 1,3-diphenylisobenzofuran (as illustrated for cycloheptyne in Fig. 3, left) was used, forming a stable oxanorbornene $(4+2)$-cycloadduct, suitable for isolation and characterization. An alternative quenching reagent for small-ring 

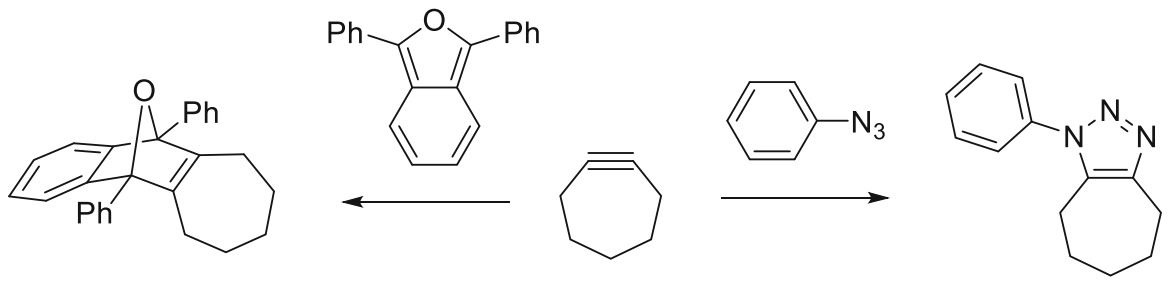

Fig. 3 Reaction of cycloheptyne with 1,3-diphenylisobenzofuran or phenyl azide, leading to bicyclic oxanorbornene (left) or phenyltriazole (right), respectively

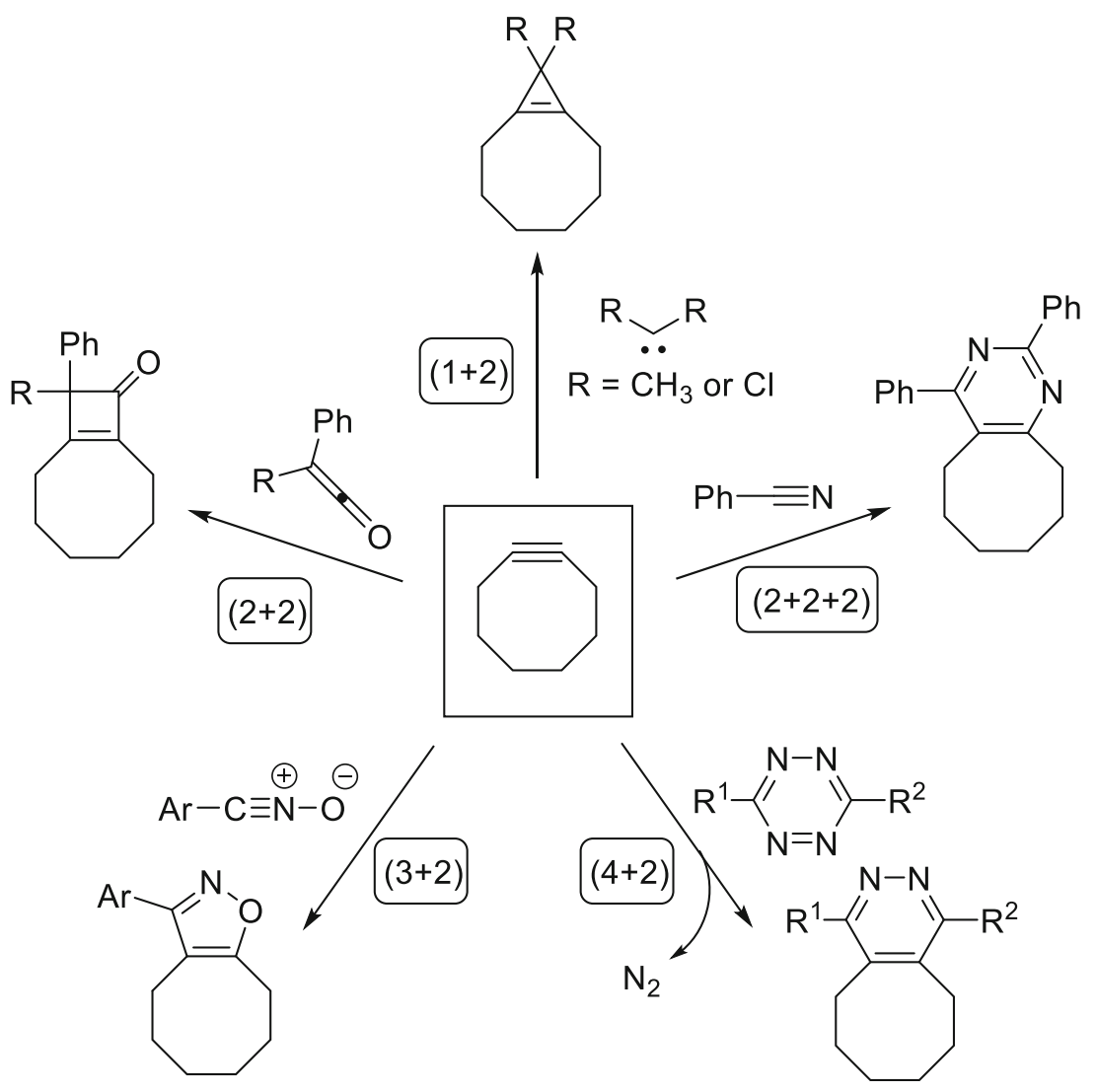

Fig. 4 Select examples of cycloaddition reactions of cyclooctyne

cycloalkynes is phenyl azide (Fig. 3, right), which will form a stable aromatic triazole by $(3+2)$-dipolar cycloaddition.

Since these first reports on the reactions of cycloalkynes with isobenzofuran and phenyl azide, a wide range of other alkynophile cycloaddition partners have become known, as conceptually illustrated with model compounds in reactions with cyclooctyne in Fig. 4. It becomes immediately clear that cycloalkynes can undergo 
a wide range of cycloaddition reactions, including $(1+2),(2+2),(2+2+2)$, $(3+2)$, and $(4+2)$ cycloadditions. Besides these, more exotic transformations like $(6+2)$ cycloadditions, hydrogen transfer reactions, radical additions, and reactions with metal salts or complexes, are also known. For further information on this topic, the reader is referred to several earlier reviews [25, 32].

\subsection{Dipolar Cycloaddition of Cycloalkynes with Azides}

The prototypical example of the reactivity of cycloalkynes in organic chemistry transformations, as already noted in the first report on the isolation of pure cyclooctyne [8], is the $(3+2)$ dipolar cycloaddition with organic azides. A logical explanation for the fast and spontaneous reaction of cycloalkynes with (phenyl)azide therefore lies in the highly favorable enthalpic release of ring-strain, by going from a strained ring to a fused ring system with favorable bond angles for the $\mathrm{sp}^{2}$-hybridized carbon atoms of the resulting triazole. It has been calculated [6] that the barrier of activation for $(3+2)$ cycloaddition is directly correlated to strain energy of cycloalkynes. Houk et al. performed calculations on the transition state of the Huisgen 1,3-dipolar cycloadditions of phenyl azide with acetylene and cyclooctyne with density functional theory at the B3LYP level [22]. The low activation energy of the cyclooctyne cycloaddition $\left(\Delta E^{*}=8.0 \mathrm{kcal} / \mathrm{mol}\right)$ compared to the strain-free acetylene cycloaddition $\left(\Delta E^{\star}=16.2 \mathrm{kcal} / \mathrm{mol}\right)$ was explained due to the decreased distortion energy for cyclooctyne to reach the requisite $\mathrm{C}-\mathrm{C}-\mathrm{C}$ bond angle of $158^{\circ}-166^{\circ}$ in the transition state versus the linear alkyne, i.e., deformation from $153^{\circ}$ and $180^{\circ}$, respectively. Given the alkyne ring strain, the reaction of an organic azide with a cyclic alkyne, typically cyclooctyne, has become commonly known as strain-promoted azide-alkyne cycloaddition (SPAAC).

\section{Speeding Up SPAAC}

\subsection{Copper-Free Click Reaction}

With most of the activity around addition reactions of cyclic alkynes taking place in the last century, interest in this particular subclass of chemistry presumably would have slowly subsided if not for the clever insight by researchers at the University of California, Berkeley [1] that strain-promoted cycloaddition of cyclooctynes with azides is a highly versatile copper-free version of the popular click reaction $[46,57]$. It was reasoned that remote attachment of a suitable functional handle to the cyclooctyne would enable the smooth conjugation to any organic azide, in any solvent of choice. In particular, it had become clear that the use of the coppercatalyzed azide-alkyne cycloaddition (CuAAC) was severely compromised in the context of biological matter, due to the toxicity of the inevitable copper(I)-species to living cells and organisms. In a seminal paper [1], it was shown that chemical functionalization of cyclooctyne with (+)-biotin enabled clean and selective visualization of living cells with azidosugars metabolically incorporated on the cell surface glycans, upon subjecting the cells to a SPAAC protocol with biotin- 
cyclooctyne followed by fluorescent labeling with a streptavidin-fluorophore. The broad impact of this seminal application of SPAAC, referred to in popular terms as "copper-free click reaction", can hardly be overestimated.

\subsection{The Quest for More Reactive Cycloalkynes}

While the first application of SPAAC rapidly found its way also outside the field of metabolic labeling, it also became quickly apparent that the relatively slow reaction kinetics required large excesses of reagents, long incubation times, and led to relatively little signal. In fact, visualization of metabolic labeling of azido-modified living cells was initially even less efficient than by Staudinger-Bertozzi ligation [49], a process known to suffer from poor reaction kinetics as well as oxygen sensitivity of the phosphine probe. As a result, a range of laboratories around the world, including ours, have embarked on the synthesis and evaluation of new cycloalkyne probes with the aim of lifting the reactivity without compromising on stability. A comprehensive overview of functionalized cyclooctynes that have been developed in the past decade, categorized by year of discovery, is provided in Fig. 5.

In general, two classes of cyclooctynes can be recognized: the earliest generation aliphatic cyclooctynes, and (di)benzoannulated cyclooctynes. The first dibenzoannulated cyclooctyne (DIBO) suitable for conjugation, developed by Ning et al. [40], has led the field to more reactive probes. It is commonly understood that the enhanced reactivity of (di)benzoannulated cyclooctynes is caused by the increase in ring strain imparted by the multiple $\mathrm{sp}^{2}$-hybridized carbons. The latter phenomenon can also account for the reactivity order BARAC $>$ DIBAC $>$ DIBO, given that the number of $\mathrm{sp}^{2}$-hybridized atoms in the ring decreases from 6 to 4 , respectively. BARAC is in fact an interesting example of the fine balancing act between reactivity and stability that comes along with the development of cyclooctyne probes: BARAC displays a reaction rate constant of nearly $1 \mathrm{~mol}^{-1} \mathrm{~s}^{-1}$ (see Table 1), but unfortunately is inherently unstable and rapidly decomposes [28]. Two cyclooctyne probes difluorobenzocycloocytne (DIFBO [55]) and 3,3,6,6-tetramethylthiaheptyne (TMTH [5]) are yet more reactive than BARAC but cannot be isolated in pure form before rapid decomposition takes place (not depicted in Fig. 5). Efforts from our own laboratory led to the development of DIBAC [16], a cyclooctyne that combined excellent stability with a reaction rate constant that is top among the family members. Interestingly, Kuzmin et al. [33] and CampbellVerduijn et al. [13] rapidly followed with publications of the same molecule, albeit obtained by different synthetic paths and with different given names (ADIBO and aza-DBCO, respectively). In this chapter, the original term DIBAC will be consistently used to denote this azacyclooctyne structure, although DBCO is also often applied.

Besides reactivity, which is typically determined in organic (co)solvents like $\mathrm{MeOH}$ or $\mathrm{MeCN}$, two other factors that qualify a given cyclooctyne, namely lipophilicity and size, are of high importance. For example, benzoannulation has shown to be beneficial for cyclooctyne reactivity but inevitably also leads to concomitant enhanced steric interactions and lipophilicity, which is typically not 


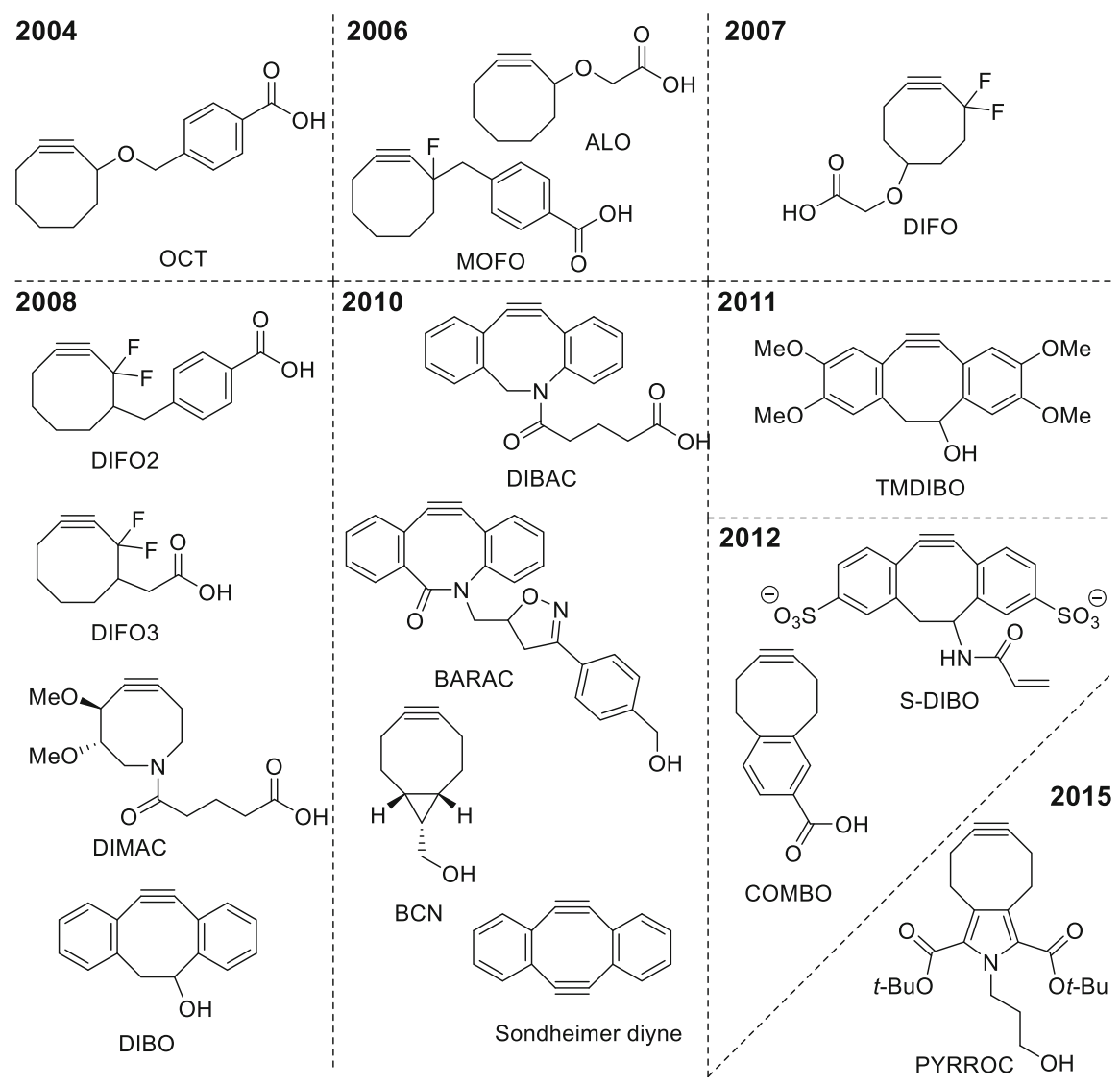

Fig. 5 Overview of functionalized cyclooctynes suitable for conjugation reactions, grouped by year of discovery. OCT cyclooctyne, $M O F O$ monofluorinated cyclooctyne, DIFO difluorocyclooctyne, DIMAC dimethoxyazacyclooctyne, $D I B O$ dibenzocyclooctyne, $D I B A C$ dibenzoazacyclooctyne, BARAC biarylazacyclooctynone, $B C N$ bicyclononyne, $T M D I B O \quad$ 2,3,6,7-tetramethoxy-DIBO, $S$-DIBO sulfonylated DIBO, COMBO carboxymethylmonobenzocyclooctyne, PYRROC pyrrolocyclooctyne

beneficial when SPAAC in water is envisaged. The first attempt to address the issue of lipophilicity was made by introduction of methoxy-groups on the cyclooctyne ring, as in DIMAC [54]. Although displaying excellent water solubility, reactivity of DIMAC was also severely compromised. Several more hydrophilic variants of DIBO have also been developed over the years, most prominently TMDIBO [56] and S-DIBO [23], both of which show much improved solubility in water, but the reaction rate constants remain rather low, as for the parent DIBO structure. The cyclooctynes COMBO [60] and PYRROC [24] have most recently been developed and are characterized by an intermediate, monobenzoannulated structure. Not unexpectedly, the reaction rate constants of COMBO and PYRROC are also lower than those for the analogous dibenzoannulated structures (Table 1). One notable exception to the rule that benzoannulation is a necessity to achieve acceptable reactivity is BCN, developed in our own laboratory [19]. For BCN, reactivity is induced 
Table 1 Reaction rate constants (for $\mathrm{BnN}_{3}$ or similar aliphatic azide) and synthetic accessibility of practically stable cyclooctynes

\begin{tabular}{llllrr}
\hline Entry & Cyclooctyne & $k\left(\times 10^{-3} \mathrm{M}^{-1} \mathrm{~s}^{-1}\right)$ & Solvent & \#Steps & Yield (\%) \\
\hline 1 & OCT & 2.4 & $\mathrm{~A}$ & 4 & 52 \\
2 & DIMAC & 3.0 & $\mathrm{~A}$ & 11 & 5 \\
3 & MOFO & 4.3 & $\mathrm{~A}$ & 5 & 15 \\
4 & PYRROC & 6.0 & $\mathrm{~A}$ & 11 & 3 \\
5 & Sondheimer & 8.8 & $\mathrm{C}$ & 4 & 41 \\
6 & DIFO2 & 42 & $\mathrm{~A}$ & 8 & 27 \\
7 & DIFO3 & 52 & $\mathrm{~A}$ & 10 & 21 \\
8 & DIFO & 76 & $\mathrm{~A}$ & 10 & 1 \\
9 & TMDIBO & 94 & $\mathrm{C}$ & 7 & 57 \\
10 & S-DIBO & 112 & $\mathrm{C}$ & 7 & 13 \\
11 & DIBO & 120 & $\mathrm{~B}$ & 5 & 10 \\
12 & BCN & 140 & $\mathrm{C}$ & 4 & 15 \\
13 & COMBO & 235 & $\mathrm{~A}$ & 6 & 11 \\
14 & DIBAC & 310 & $\mathrm{C}$ & 9 & 41 \\
15 & BARAC & 960 & $\mathrm{~A}$ & 6 & 18 \\
\hline
\end{tabular}

Cyclooctynes are listed in order of reactivity. Solvent: $A=\mathrm{CD}_{3} \mathrm{CN}, \mathrm{B}=\mathrm{CD}_{3} \mathrm{CN}: \mathrm{D}_{2} \mathrm{O}$ (3:1), $\mathrm{C}=\mathrm{CD}_{3} \mathrm{OD}$ or $\mathrm{CH}_{3} \mathrm{OH}$

by ring fusion of cyclooctyne to cyclopropane, leading to the typical bicyclo[6.1.0]non-4-yne structure. Although less reactive than DIBAC, reaction rate constants for the endo-isomer are still among the highest in the pack (see Table 1), while the synthesis of $\mathrm{BCN}$ is exceptionally short and simple.

From Fig. 5, it also becomes apparent that while active development of cyclooctynes took place in the years 2008-2010, the intensity in the field has more or less subsided in the past years. One possible reason for this observation may be found in the fact that further boosting of the reactivity of cyclooctyne for azide is typically penalized by loss in stability, as earlier mentioned for BARAC, DIFBO, and TMTH. Another explanation lies in the current commercial availability of the cyclooctynes DIBO, DIBAC, and $\mathrm{BCN}$, the three of which have dominated the field of SPAAC in the past years.

\subsection{Influence of Azide Structure on Reaction Rate}

While significant effort has been devoted over the years to the development of more reactive cyclooctynes, as delineated above, only scant investigations so far have focused on the increase of SPAAC rates by modulation of the complementary component, i.e., the azide. In fact, the vast majority of reported applications of SPAAC are based on reaction with simple aliphatic azides. As a logical consequence, reaction rate constants are also nearly always determined with an aliphatic azide (typically benzyl azide), but seldom with an aryl azide. One possible reason that aromatic azides are generally avoided for SPAAC may lie in a report 


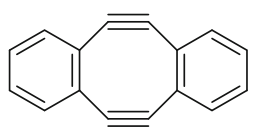

Sondheimer diyne

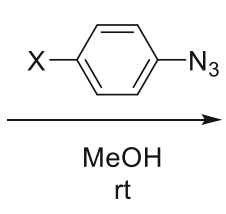

$\mathrm{rt}$

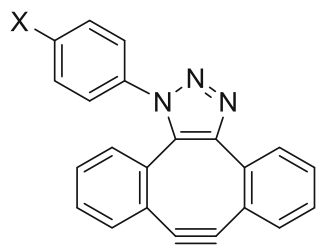

\begin{tabular}{|c|c|}
\hline $\mathrm{X}$ & $\mathrm{k}_{\text {rel }}$ \\
\hline $\mathrm{H}$ & 1 \\
$\mathrm{OMe}$ & 3.8 \\
$\mathrm{CF}_{3}$ & 0.9 \\
\hline
\end{tabular}

Fig. 6 Negligible influence of azidobenzene para-substitution on reaction rate with dibenzoannulated cyclooctyne

that $p$-azidophenylalanine shows sevenfold lower reactivity than that of the corresponding aliphatic azidomethyl analogue [66], at least in conjunction with DIBAC, a dibenzoannulated cyclooctyne. Furthermore, the observation that reaction rates of aromatic azides are hardly influenced by changing the electronic nature of substituents (as determined for $p$-methoxy and $p-\mathrm{CF}_{3}$-phenyl azide, see Fig. 6) may have provided further ground to avoid aromatic azides for SPAAC [64]. These observations obviously support the notion that aryl azides, and in particular electron-poor azides (as in $p-\mathrm{CF}_{3}-\mathrm{PhN}_{3}$ ), are better avoided in case high SPAAC reaction rates are desirable. Other studies have reported a reactivity enhancement (up to $2.2 \times$ faster) upon the introduction of electron-withdrawing halogen substituents on DIFO [4], BARAC [28] and DIBAC [14, 18], all of which suggests that the SPAAC mechanism primarily proceeds via $\mathrm{HOMO}_{\text {azide }}-\mathrm{LUMO}_{\text {cyclooctyne }}$ interaction so that electron-rich azides are preferred. Interestingly, it has been known since the 1960s that electronegative substituents on aryl azides accelerate reaction rates with strained alkenes, for example a fourfold reaction rate enhancement of $p$-nitro substitution of phenyl azide with norbornene [50]. Even more markedly, picryl azide was found to react with norbornene almost 1000 times faster than phenyl azide [2].

Based on these observations, we recently concluded that the apparent slower reaction of electron-poor azides in SPAAC only holds for benzoannulated cyclooctynes (with low-lying LUMO), while in combination with more electronrich cyclooctynes (like $\mathrm{BCN}$ ), azides can react by a second, inverse electrondemand mechanism (i.e., SPAAC) as well [20]. As a result, it was first found that strain-promoted cycloaddition of aromatic azides with $\mathrm{BCN}$ is nearly eight times faster than with DIBAC, which is the opposite trend for reaction with benzyl azide. Introduction of electron-withdrawing substituents on the aryl azide led to a further acceleration in reaction with $\mathrm{BCN}$, while reaction rate with DIBAC stayed more or less constant. The highest reaction rate acceleration was achieved with a pyridinium derivative, giving an almost 30 -fold by faster reaction than benzyl azide and an absolute rate constant of almost $2 \mathrm{M}^{-1} \mathrm{~s}^{-1}$ (Fig. 7).

\subsection{Solvent Effects}

Since Breslow first pointed out the effect of water on cycloaddition reactions [45], an extensive amount of kinetic measurements in the field have been determined, in particular focusing on the Diels-Alder reaction, a $(4+2)$-cycloaddition reaction. Studies of the comparative rates of 1,3-dipolar cycloaddition reactions in water and 


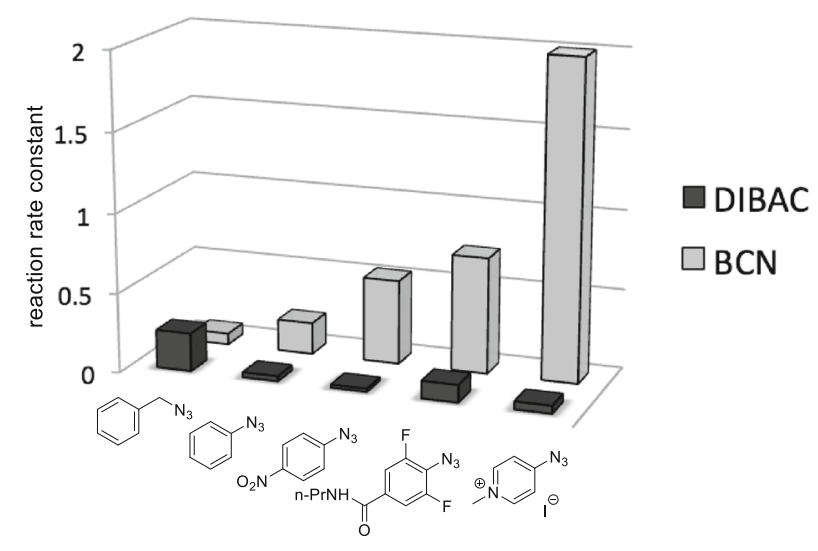

Fig. 7 Relationship between nature of the azide, structure of cyclooctyne and reaction rate constants. Reaction rate constants were determined in $\mathrm{CD}_{3} \mathrm{CN}: \mathrm{D}_{2} \mathrm{O}=3: 1$

organic solvents have not attracted the same level of attention. Some work in the field has focused on reactions of transient nitrile oxides [59] and nitrilimines [39] generated in aqueous environments and there are some reports on rate measurements on 1,3dipolar cycloaddition reactions in water-organic mixtures. Recently, it was reported that with an increasing mole fraction of water, significant enhancement of 1,3-dipolar cycloaddition rates occurs [12]. It was suggested that a dominant hydrogen-bonding effect operates in water-induced rate enhancements of 1,3-dipolar cycloaddition reactions. The hydrogen-bonding effect involves secondary hydrogen bond bridging from the primary water-solvation shell of the transition state and the growth of structured water clusters, which was also supported by theoretical calculations.

The awareness that increasing levels of water translate into faster reaction rates has received surprisingly little attention in the field of SPAAC, despite the fact that a large number of applications involve conjugation processes on natural biomolecules (peptides, proteins, glycans, oligonucleotides) in aqueous systems. Nevertheless, classification and appreciation of a given cyclooctyne probe is typically only performed by determination of reaction rate constants in any of a range of solvents like methanol, acetonitrile or water (or deuterated versions thereof if performed in NMR, vide infra), or mixtures thereof. For example, Table 1 above displays the reaction rate constants of the known cyclooctynes across these solvents, which in fact makes it difficult to truly compare the usefulness of these probes in a quantifiable manner. Furthermore, it has been noted by several authors that SPAAC proceeds (significantly) faster in more aqueous solvent systems [9, 19, 24, 40, 60].

For these reasons, we embarked on a study to determine the reaction rate constants of the most commonly applied dibenzoannulated and aliphatic cyclooctynes, respectively DIBAC and BCN, as deduced from a SciFinder ${ }^{\circledR}$ structure search of these compounds. As becomes clear, since the year of its inception (2010), DIBAC has made its appearance in 260 unique publications (184 scientific publications and 80 patent applications) while BCN is reported 155 times (103 papers and 51 patent application). For comparison, DIBO, the third-most popular cyclooctyne gave 135 
unique hits for the same time-frame (74 papers, 42 patent applications). Moreover, given the lower reaction rate constant of DIBO versus DIBAC, as well as their large structural similarity, DIBO was omitted from the studies below.

It was decided to evaluate both $\mathrm{DIBAC}$ and $\mathrm{BCN}$ with a range of representative azides and in three different solvent systems: $\mathrm{MeOD}, \mathrm{CD}_{3} \mathrm{CN}: \mathrm{D}_{2} \mathrm{O}$ (1:3), both for NMR measurements, and THF: $\mathrm{H}_{2} \mathrm{O}$ (9:1), for quantification by IR. In order to ensure that both probes and azides would be soluble in the acetonitrile-water mixture, a hydroxyethylated derivative was prepared in several instances. The same strategy was applied to some of the more lipophilic aromatic azides, which led to full solubility except for the diisopropylated azidobenzene derivative (entry 5), where NMR was performed in a $\mathrm{MeCN}: \mathrm{H}_{2} \mathrm{O}$ (1:2) mixture.

Several interesting observations can be made from Table 2. First of all, the picture is confirmed that DIBAC is faster than $\mathrm{BCN}$ in reaction with aliphatic azides (entries 1-3). While apparent in all cases, the reaction rate difference is markedly larger in MeOD or $\mathrm{CD}_{3} \mathrm{CN}: \mathrm{D}_{2} \mathrm{O}$ (factor $\sim 10$ ) than in THF: $\mathrm{H}_{2} \mathrm{O}$ (factor $\sim 3$ ). Also striking is the rate constant of $1.9 \mathrm{M}^{-1} \mathrm{~s}^{-1}$ for reaction of DIBAC with benzyl azide (entry 1), which is more than double than that for the other aliphatic azides (entries $2+3$ ). We attribute this to the relatively low solubility of benzyl azide in an aqueous solvent system, resulting in faster reaction by means of a hydrophobic effect. The hydrophobic effect may also be accountable for another interesting observation, that the reactions of DIBAC with aliphatic azides are much faster in $75 \%$ aqueous acetonitrile than in methanol or in $10 \%$ aqueous THF.

As expected, the reactivity trend is reversed for azidobenzene (entry 4), where $\mathrm{BCN}$ is up to seven times faster than DIBAC (in $75 \%$ aqueous $\mathrm{CD}_{3} \mathrm{CN}$ ), with a reaction a rate constant of $0.75 \mathrm{M}^{-1} \mathrm{~s}^{-1}$. As reported earlier [64], introduction of an ortho-isopropyl group on azidobenzene (entry 5) leads to significant rate acceleration for reaction with DIBAC, which is already very high in $\operatorname{MeOD}\left(2.3 \mathrm{M}^{-1} \mathrm{~s}^{-1}\right)$ and too fast to measure in $\mathrm{D}_{2} \mathrm{O}: \mathrm{CD}_{3} \mathrm{CN}$ by NMR. The number provided in the Table $\left(\sim 4 \mathrm{M}^{-1} \mathrm{~s}^{-1}\right)$ for this solvent system is instead derived by multiplying the rate constant determined for $\mathrm{BCN}\left(1.5 \mathrm{M}^{-1} \mathrm{~s}^{-1}\right)$ by the earlier determined relative reaction rate DIBAC: $\mathrm{BCN}=2.5$ [20]. A similar strategy estimates the reaction rate constant of BCN with the electron-poor difluorinated phenylazide $\left(\sim 6 \mathrm{M}^{-1} \mathrm{~s}^{-1}\right.$, entry 6) from the value experimentally determined for DIBAC.

\subsection{Tools to Quantify SPAAC Reaction Rates}

The main determinant of the quality of any given cyclooctyne for SPAAC reaction is its reaction rate constant with azide (aliphatic or aromatic). Throughout the years, a large number of reaction rate constants have been experimentally determined for different cycloalkynes and azides, mainly by four analytical techniques: (1) NMR, (2) UV spectroscopy, (3) IR spectroscopy, and (4) fluorescence.

The most commonly applied method to determine a SPAAC reaction rate constant is by NMR [1]. To this end, a cyclooctyne and an azide are mixed in a deuterated solvent and formation of product is quantified by integration of diagnostic peaks of the formed triazole product. Given the fact that the triazole ring itself is fully substituted, other diagnostic protons in the product with a unique, non- 


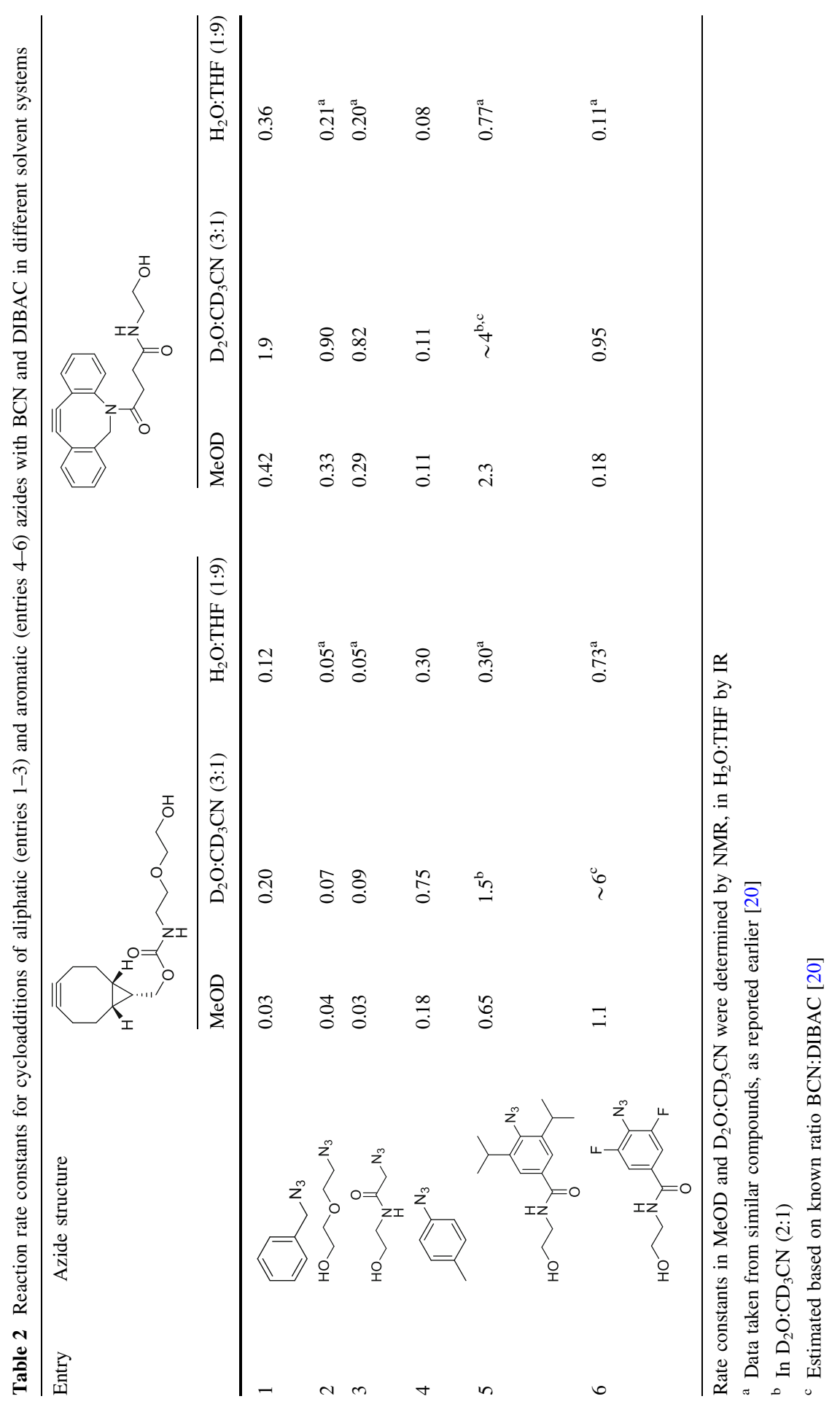


overlapping shift in the NMR spectrum, must be identified (or specifically introduced in one of the substrates). Alternatively, one or more distinct proton peaks of starting material can be integrated. It must be noted that the formation of a mixture of regioisomeric triazoles, as is typical for all the cyclooctynes except the $\mathrm{C}_{2}$-symmetric versions, is often a complicating factor. From the conversion plots thus obtained, the second-order rate plots can be calculated according to Eq. (1):

$$
k t=\frac{1}{[B]_{0}-[A]_{0}} \times \ln \frac{[A]_{0}\left([B]_{0}-[P]\right)}{\left([A]_{0}-[P]\right)[B]_{0}}
$$

with $k=$ second-order rate constant $\left(\mathrm{M}^{-1} \mathrm{~s}^{-1}\right), t=$ reaction time $(\mathrm{s}),[A]_{0}=$ the initial concentration of substrate $\mathrm{A}(\mathrm{mmol} / \mathrm{ml}),[B]_{0}=$ the initial concentration of substrate $\mathrm{B}(\mathrm{mmol} / \mathrm{ml})$ and $[P]=$ the concentration of triazole product $(\mathrm{mmol} / \mathrm{ml})$. Either cyclooctyne or azide can be applied in excess, preferably between 1.2 and 2 (but not stoichiometric). By plotting $t$ versus $k t$, calculation of the slope of the resulting (straight) line, gives the reaction rate constant $k$. Better plots are obtained by only including data points up to approximately $50 \%$ conversion. Given the relatively high required concentration of components for fast NMR measurements (typically $10 \mathrm{mM}$ or higher) and the rather time-consuming process before the first measurement can take place (mixing of reagents, insertion in magnet, shimming, scanning), it is clear that NMR has its limitations for very fast SPAAC $\left(>0.5 \mathrm{M}^{-1} \mathrm{~s}^{-1}\right)$, because more than $50 \%$ of starting material may already be converted at the first measurement and few data points can be taken.

Two alternative techniques for real-time monitoring of (fast) SPAAC processes are UV and IR spectroscopy. For example, rate measurements of SPAAC can be conducted by reaction of a 10-100-fold excess of azide to a low concentration of acetylene (down to $1 \times 10^{-4} \mathrm{M}$ ) in $\mathrm{MeOH}$. Reaction in this case can be monitored by following the decay of the characteristic absorbance of the acetylene bond, as for example for dibenzoannulated cyclooctyne DIBO [43]. This method may give more accurate rate constants compared to the use of NMR, especially for fast reactions. Importantly, with this large excess of azide over cyclooctyne, the UV spectroscopic method is performed under pseudo-first-order conditions over a wide range of reagent concentrations, making the analysis of second-order kinetic curves more reliable. In case the UV absorption of the acetylene bond is less distinct, as for the aliphatic cyclooctynes, and no other specific UV chromophores can be identified in starting materials or reagents, an IR-based method may be a viable alternative. We recently reported [20] that the substrate-to-product conversion can be directly monitored by integration of the distinct stretch vibration of azide $\left(\sim 2100 \mathrm{~cm}^{-1}\right)$. Conveniently, deuterated solvents are not necessary and it was found that IR monitoring could even be executed in $10 \%$ aqueous THF.

The most sensitive method for determination of reaction rate constants of cyclooctynes is by means of a reaction with a fluorogenic azide substrate [35]. By definition, a fluorogenic SPAAC process involves a reaction between a nonfluorescent alkyne and azide, allowing the ligation of two biomolecules to afford a highly fluorescent triazole product. Besides that, compounds that become fluorescent upon reaction with a chemical reporter and without the need of copper have 
many attractive features as bioorthogonal probes, such as eliminating the need for probe washout, reducing background labeling, and offering opportunities for monitoring biological processes in real time. The first fluorogenic click reaction based on readily synthesized azidocoumarin derivatives was reported by Sivakumar et al. [53]. Moreover, the photophysical fluorescent properties of coumarines can be strongly enhanced by substitution with an electron withdrawing group at the 3-position and/or an electron donating group at the 7-position. Other early variants of fluorogenic azides involve azidomethylated 1,8-naphthalimide dyes [48] and fluorogenic dyes based on a photoinduced electron transfer (PET) process with anthracene as a fluorophore [63]. It must be noted that the vast majority of fluorogenic probes feature an aromatic azide, which obviously will have a major impact on determination of reaction rate constant in absolute terms, as was for example found by us for a range of BCN derivatives [36]. Other more recent fluorogenic azides, as developed by Shieh et al. [51] and Herner et al. [26] and even a fluorogenic azide substrate for generation of a near-infrared (NIR) triazole dye [52], are depicted in Fig. 8.

Notwithstanding the elegancy and versatility of the azide-based fluorogenic probes, in particular to enable the visualization of biomolecules in living systems<smiles>Nc1cc2ccc(O)cc2oc1=O</smiles>

Sivakumar et al 2004<smiles>CCN(CC)c1ccc2cc(/C=C/c3ccc(N)cc3)oc2c1</smiles><smiles>CCN1C(=O)c2cccc3c([N+](=O)[O-])ccc(c23)C1=O</smiles>

06<smiles>[R]c1c2ccccc2c(CN)c2ccccc12</smiles>

Xie et al 2008

Herner et al 2014<smiles>Nc1ccc(-c2c3ccc(=O)cc-3oc3cc(O)ccc23)c2ccccc12</smiles>

Shieh et al 2012<smiles>C[N+]=C1C=CC2=C(c3cc([N+])c(OC)cc3OC)c3ccc(N(C)C)cc3[Si](C)(C)C2=C1</smiles>

Shieh et al 2014

Fig. 8 Fluorogenic azide probes for cycloaddition with alkynes 
<smiles>[R]c1cc(=O)oc2cc(C#C)ccc12</smiles>

Zhou et al 2004<smiles>CN1C(=O)c2ccccc2C#Cc2cc3oc(=O)cc(-c4ccccc4)c3cc21</smiles>

Jewett and Bertozzi 2011<smiles>COc1ccc2c(c1)C#Cc1cc(OC)ccc1-c1c-2c1=O</smiles>

Friscourt et al 2012<smiles>[R10][Y17]NCC(=O)Nc1ccc(-c2nnc(-c3ccccn3)nn2)nc1</smiles>

Lang et al 2012

Fig. 9 Fluorogenic alkynes for cycloaddition with azides

with biooorthogonal chemistry, it is also clear that for fluorescence detection of an azide label in a biomolecular environment, a complementary fluorogenic alkyne structure is desired. The earliest example of the latter (Fig. 9) was provided in the form of acetylenic derivatives of coumarin by Zhou and Fahrni [65], highly analogous to the azido-coumarin derivatives described by Sivakumar et al. [53]. However, clearly the reaction of a terminal alkyne with an azide requires the undesirable presence of copper(I) to induce the formation of triazole. Two fluorogenic cyclooctyne versions have been developed in the past few years. The first probe was based on annulation of coumarin to BARAC and was developed by Jewett and Bertozzi [29]. Secondly, Friscourt et al. [23] reported that the cyclopropenone derivative of Sondheimer diyne unexpectedly forms a highly fluorescent triazole upon a copper-free reaction with azide. Most recently, Lang et al. [34] showed that a TAMRA-functionalized tetrazine derivative leads to fivefold to tenfold increase in fluorescence signal, similar to earlier reported for reaction with trans-cyclooctene and norbornene, upon reaction with $\mathrm{BCN}$.

\section{Concluding Remarks and Future Prospects}

Strain-promoted azide-alkyne cycloaddition (SPAAC), since its inception in 2004, has firmly established itself as a powerful click chemistry tool. The commercial access of starting materials, its ease of operation, the nowadays practical reaction 
rates, even under high dilution conditions, and the stability of the resulting triazole product, have catapulted SPAAC at the forefront in many research areas in academia and industry. Originally developed for application in bioorthogonal chemistry, SPAAC has proven its value more and more in additional areas of science such as bioconjugation processes, hybrid and block polymers, highperformance and self-regenerative materials, metabolic engineering of biological systems and beyond. One key to the success of SPAAC is the azide component in the reaction, which is easily accessible, small and stable [17]. Nevertheless, recent years have elegantly demonstrated the power of cyclooctyne chemistry beyond cycloaddition with organic azides. Although much of these reactions were known for more than 50 years, mostly by work of Huisgen in the field of 1,3-dipolar cycloadditions, the past decade has witnessed a strong revival of cyclooctyne for development of fast and selective reaction with a range of other alkynophiles. For example, we were to apply [41] strain-promoted alkyne-nitrone cycloaddition (SPANC), a reaction also reported by McKay et al. in the same year [37], for the N-terminal labeling of proteins. Similarly, both our laboratory [27] and Sanders et al. [47] reported the reaction of cyclooctynes with nitrile oxides (SPANOC) leading to isoxazoles, which is a factor $\sim 10$ faster than SPAAC and SPANC. Finally, strain-promoted cycloaddition with diazo-compounds is also known [47] as well as reaction of BCN with sydnone [42, 61]. Interestingly, in the field of $(4+2)$ cycloadditions, cyclooctynes show reaction rate constants more than a factor 1000 higher than for 1,3-dipolar cycloadditions. It has been known for more than 30 years that aliphatic cyclooctynes undergo extremely fast $(4+2)$ cycloadditions with electron-poor tetrazines [3]. The latter process can be tailored to specific reaction rates with electron-rich cyclooctynes like $\mathrm{BCN}$ in organic solvents [15] and was found to proceed at a surprisingly high reaction rates $>1000 \mathrm{M}^{-1} \mathrm{~s}^{-1}$ under aqueous conditions when applied for protein labeling [9, 34]. We most recently showed that $(4+2)$ cycloaddition of BCN with 1,2-quinone (SPOCQ) proceeds with reaction rates intermediate of azide and tetrazine $\left(\sim 500 \mathrm{M}^{-1} \mathrm{~s}^{-1}\right)$ and can be applied for rapid labeling of proteins with genetically encoded BCN [10] or formation of gel networks in the order of seconds [30].

Finally, it seems fair to state that SPAAC has now matured into more than just a spectacular click reaction or exciting research tool. As noted earlier, only in the past 5 years more than 100 patent applications have been filed on the use of SPAAC with DIBAC or BCN, with applications in e.g., nanoparticle functionalization, polymer functionalization, genetic encoding, (biodegradable) hydrogels, controlled drug release, oligonucleotide tagging, DNA libraries, peptide arrays, long-acting biopharmaceuticals, radioisotope labeling, and nuclear imaging. Among these, arguably the most prominent application of SPAAC is in the field of the selective and site-specific conjugation of proteins, such as PEGylation, radioisotope labeling, and controlled release of biopharmaceuticals. For example, in the field of targeted anti-cancer therapy alone, a range of pharmaceutical companies (MedImmune, Novartis, Agensys, Sutro, Innate Pharma, Synaffix) are building (part of) their technology around the controlled attachment of highly potent toxins (payloads) to monoclonal antibodies - to prepare antibody-drug conjugates or ADCs-by means of installation of azide into the protein (genetic encoding or enzymatically) followed 
by highly specific SPAAC. A remarkable position for the process of spontaneous cycloaddition of cyclooctyne and azide, discovered more than 60 years ago out of purely chemical curiosity.

Open Access This article is distributed under the terms of the Creative Commons Attribution 4.0 International License (http://creativecommons.org/licenses/by/4.0/), which permits unrestricted use, distribution, and reproduction in any medium, provided you give appropriate credit to the original author(s) and the source, provide a link to the Creative Commons license, and indicate if changes were made.

\section{References}

1. Agard NJ, Prescher JA, Bertozzi CR (2004) A strain-promoted [3 + 2] azide-alkyne cycloaddition for covalent modification of biomolecules in living systems. J Am Chem Soc 126:15046-15047

2. Bailey AS and White JE (1966) Reaction of picryl azide with olefins. J Chem Soc B 819-822

3. Balcar J, Chrisam G, Huber FX, Sauer J (1983) Reactivity of nitrogen-heterocycles with cyclooctyne as dienophile. Tetrahedron Lett 24:1481-1484

4. Baskin JM, Prescher JA, Laughlin S et al (2007) Copper-free click chemistry for dynamic in vivo imaging. Proc Natl Acad Sci USA 104:16793-16797

5. De Almeida G, Sletten EM, Nakamura H, Palaniappan KK, Bertozzi CR (2012) Thiacycloalkynes for Copper-Free Click Chemistry. Angew Chem Int Ed 51:2443-2447

6. Bach RD (2009) Ring strain energy in the cyclooctyl system. The effect of strain energy on [3 +2$]$ cycloaddition reactions with azides. J Am Chem Soc 131:5233-5243

7. Blomquist AT, Burge RE Jr, Liu LH et al (1951) Many-membered carbon rings. IV. Synthesis of cyclononyne and cyclodecyne. J Am Chem Soc 73:5510-5511

8. Blomquist AT, Liu LH (1953) Many-membered carbon rings. VII. Cyclooctyne. J Am Chem Soc 75:2153-2154

9. Borrmann A, Milles S, Plass T (2012) Genetic encoding of a Bicyclo[6.1.0]nonyne-charged amino acid enables fast cellular protein imaging by metal-free ligation. ChemBioChem 13:2094-2099

10. Borrmann A, Fatunsin O, Dommerholt $J$ et al (2015) Strain-promoted oxidation-controlled cyclooctyne-1,2-quinone cycloaddition (SPOCQ) for fast and activatable protein conjugation. Bioconj Chem 26:257-261

11. Brandsma L, Verkruijsse HD (1978) An improved synthesis of cyclooctyne. Synthesis. 290

12. Butler RN, Cunningham WJ, Coyne AG, Burke LA (2012) The influence of water on the rates of 1,3dipolar cycloaddition reactions: trigger points for exponential rate increases in water-organic solvent mixtures. water-super versus water-normal dipolarophiles. J Am Chem Soc 126:11923-11929

13. Campbell-Verduijn LS, Mirfeizi L, Schoonen AK, Dierckx RA, Elsinga PH, Feringa BL (2011) Strain-promoted copper-free "Click" Chemistry for ${ }^{18} \mathrm{~F}$ radiolabeling of Bombesin. Angew Chem Int Ed 123:11313-11316

14. Chadwick RC, Van Gyzen S, Liogier S, Adronov A (2014) Scalable synthesis of strained cyclooctyne derivatives. Synthesis 46:669-677

15. Chen W, Wang D, Dai C et al (2012) Clicking 1,2,4,5-tetrazine and cyclooctynes with tunable reaction rates. Chem Commun 48:1736-1738

16. Debets MF, van der Doelen CWJ, Rutjes FPJT et al (2010) Azide: a unique dipole for metal-free bioorthogonal ligations. ChemBioChem 11:1168-1184

17. Debets MF, van Berkel SS, Schoffelen S et al (2010) Aza-dibenzocyclooctynes for fast and efficient enzyme PEGylation via copper-free $(3+2)$ cycloaddition. Chem Commun 46:97-99

18. Debets MF, Prins JS, Merckx D et al (2014) Synthesis of DIBAC analogues with excellent SPAAC rate constants. Org Biomol Chem 12:5031-5037

19. Dommerholt J, Schmidt S, Temming R et al (2010) Readily accessible bicyclononynes for bioorthogonal labeling and three-dimensional imaging of living cells. Angew Chem Int Ed 49:9422-9425

20. Dommerholt J, van Rooijen O, Borrmann A et al (2014) Highly accelerated inverse electron-demand cycloaddition of electron-deficient azides with aliphatic cyclooctynes. Nature Commun 5:5378 
21. Domnin NA (1938) The triple bond in carbon rings and the probable structure of the simplest cyclic hydrocarbon of the composition $\mathrm{C}_{\mathrm{n}} \mathrm{H}_{2 \mathrm{n}-4}$. II. Synthesis of cyclooctyne. J Gen Chem USSR 8:851-868

22. Ess DH, Jones GO, Houk KN (2008) Transition states of strain-promoted metal-free click chemistry: 1,3-dipolar cycloadditions of phenyl azide and cyclooctynes. Org Lett 10:1633-1636

23. Friscourt F, Ledin PA, Mbua NE et al (2012) Polar dibenzocyclooctynes for selective labeling of extracellular glycoconjugates of living cells. J Am Chem Soc 134:5381-5389

24. Gröst C, Berg T (2015) PYRROC: the first functionalized cycloalkyne that facilitates isomer-free generation of organic molecules by SPAAC. Org Biomol Chem 13:3866-3870

25. Heber D, Rösner P, Tochtermann W (2005) Cyclooctyne and 4-cyclooctyn-1-ol—versatile building blocks in organic synthesis. Eur J Org Chem 4231-4247

26. Herner A, Girona GA, Nikíc I (2014) New generation of bioorthogonally applicable fluorogenic dyes with visible excitations and large stokes shifts. Bioconj Chem 25:1370-1374

27. Jawalekar AM, Reubsaet E, Rutjes FPJT et al (2011) Synthesis of isoxazoles by hypervalent iodineinduced cycloaddition of nitrile oxides to alkynes. Chem Commun 47:3198-3200

28. Jewett JC, Sletten EM, Bertozzi CR (2010) Rapid cu-free click chemistry with readily synthesized biarylazacyclooctynones. J Am Chem Soc 132:3688-3690

29. Jewett JC, Bertozzi CR (2011) Synthesis of a fluorogenic cyclooctyne activated by cu-free click Chemistry. Org Lett 13:5937-5939

30. Jonker AM, Borrmann A, van Eck ERH et al (2015) A fast and activatable cross-linking strategy for hydrogel formation. Adv Mat 27:1235-1240

31. Krebs A, Kimling H (1971) [1 + 2] cycloadditions of isocyanides to alkynes. Angew Chem Int Ed 10:409-410

32. Krebs A, Wilke J (1983) Angle strained cycloalkynes. Top Curr Chem 109:189-233

33. Kuzmin A, Poloukhtine A, Wolfert MA, Popik VV (2010) Surface functionalization using catalystfree azide-alkyne cycloaddition. Bioconj Chem 21:2076-2085

34. Lang K, Davis L, Wallace S et al (2012) Genetic encoding of bicyclononynes and trans-cyclooctenes for site- specific protein labeling in vitro and in live mammalian cells via rapid fluorogenic DielsAlder reactions. J Am Chem Soc 134:10317-10320

35. Le Troumaguet C, Wang C, Wang Q (2010) Fluorogenic click reaction. Chem Soc Rev 39:1233-1239

36. Leunissen EHP, Meuleners MHL, Verkade JMM et al (2014) Copper-free click with polar BCN derivatives for modulation of cellular imaging. ChemBioChem 15:1445-1451

37. McKay CS, Moran J, Pezacki JP (2010) Nitrones as dipoles for rapid strain-promoted 1,3-dipolar cycloadditions with cyclooctynes. Chem Commun 46:931-933

38. Meier H (1972) Synthesis of medium-sized cycloalkynes. Synthesis 235-253

39. Molteni G, Orlandi M, Broggini G (2000) Nitrilimine cycloadditions in aqueous media. J Chem Soc Perkin Trans 1:3742-3745

40. Ning X, Guo J, Wolfert MA, Boons GJ (2008) Visualizing metabolically labeled glycoconjugates of living cells by copper-free and fast Huisgen cycloadditions. Angew Chem Int Ed 47:2253-2255

41. Ning X, Temming RP, Dommerholt J et al (2010) Protein modification by strain-promoted alkynenitrone cycloaddition. Angew Chem Int Ed 49:3065-3068

42. Plougastel L, Koniev O, Specklin S et al (2014) 4-Halogeno-sydnones for fast strain-promoted cycloaddition with bicyclo-[6.1.0]-nonyne. Chem Commun 50:9376-9378

43. Poloukthine AA, Mbua NE, Wolfert MA et al (2009) Selective labeling of living cells by a phototriggered click reaction. J Am Chem Soc 131:15770-15776

44. Prelog V, Schenker K, Küng W (1953) Zur Kenntnis des Kohlen-stoffringes. 62. Mitteilung. Zur Kenntnis des Neuringes. Über die transanulare Oxydation der Cyclonene zu Cyclononandiolen-(1,5). Helv Chim Acta 36:471-482

45. Rideout DC, Breslow RJ (1980) Hydrophobic acceleration of Diels-Alder reactions. J Am Chem Soc 102:7816-7817

46. Rostovtsev VV, Green LG, Fokin VV et al (2002) A stepwise Huisgen cycloaddition process: copper(I)-catalyzed regioselective "ligation" of azides and terminal alkynes. Angew Chem Int Ed 41:2596-2599

47. Sanders BC, Friscourt F, Ledin PA et al (2011) Metal-free sequential [3 + 2]-dipolar cycloadditions using cyclooctynes and 1,3-dipoles of different reactivity. J Am Chem Soc 133:949-957

48. Sawa M, Hsu TL, Itoh T et al (2006) Glycoproteomic probes for fluorescent imaging of fucosylated glycans in vivo. Proc Nat Acad Sci USA 103:12371-12376 
49. Saxon E, Bertozzi CR (2000) Cell surface engineering by a modified Staudinger reaction. Science 287:2007-2010

50. Scheiner P, Schomaker JH, Deming S et al (1965) The addition of aryl azides to norbornene. A kinetic investigation. J Am Chem Soc 87:306-311

51. Shieh P, Hangauer MJ, Bertozzi CR (2012) Fluorogenic azidofluoresceins for biological imaging. J Am Chem Soc 134:17428-17431

52. Shieh P, Sloan Siegrist M, Cullen AJ et al (2014) Imaging bacterial peptidoglycan with near-infrared fluorogenic azide probes. Proc Nat Acad Sci 111:5456-5461

53. Sivakumar K, Xie F, Cash BM et al (2004) A fluorogenic 1,3-dipolar cycloaddition reaction of 3-Azidocoumarins and acetylenes. Org Lett 6:4603-4606

54. Sletten EM, Bertozzi CR (2008) A hydrophilic azacyclooctyne for Cu-free click Chemistry. Org Lett 10:3097-3099

55. Sletten EM, Nakamura H, Jewett JC et al (2010) Difluorobenzocyclooctyne: synthesis, reactivity, and stabilization by $\beta$-cyclodextrin. J Am Chem Soc 132:11799-11805

56. Stöckmann H, Neves AA, Stairs S et al (2011) Development and evaluation of new cyclooctynes for cell surface glycan imaging in cancer cells. Chem Sci 2:932-936

57. Tornøe CW, Christensen C, Meldal M (2002) Peptidotriazoles on solid phase: [1,2,3]-triazoles by regiospecific Copper(I)-catalyzed 1,3-dipolar cycloadditions of terminal alkynes to azides. J Org Chem 67:3057-3064

58. Turner RB, Jarrett AD, Goebel P et al (1973) Heats of hydrogenation. IX. Cyclic acetylenes and some miscellaneous olefins. J Am Chem Soc 95:790-792

59. Van Mersbergen D, Wijnen JW, Engberts JBFN (1998) 1,3-dipolar cycloadditions of benzonitrile oxide with various dipolarophiles in aqueous solutions. A kinetic study. J Org Chem 63:8801-8805

60. Varga BR, Kállay M, Hegyi K et al (2012) A non-fluorinated monobenzocyclooctyne for rapid Copper-free click reactions. Chem Eur J 18:822-828

61. Wallace S, Chin JW (2014) Strain-promoted sydnone bicyclo-[6.1.0]-nonyne cycloaddition. Chem Sci 5:1742-1744

62. Wittig G, Krebs A (1961) On the existence of small-membered cycloalkynes, I. Chem Ber 94:3260-3275

63. Xie F, Sivakumar K, Zeng Q et al (2008) A fluorogenic $\mathrm{Cu}(\mathrm{I})$ catalyzed azide-alkyne cycloaddition reaction of azidoanthracene derivatives. Tetrahedron 64:2906-2914

64. Yoshida S, Shiraishi A, Kanno K et al (2011) Enhanced clickability of doubly sterically-hindered aryl azides. Sci Rep 82:1-4

65. Zhou Z, Fahrni CJ (2004) A fluorogenic probe for the Copper(I)-catalyzed azide-alkyne ligation reaction: modulation of the fluorescence emission via ${ }^{3}\left(n, \pi^{*}\right)-^{1}\left(\pi, \pi^{*}\right)$ inversion. J Am Chem Soc 126:8862-8863

66. Zimmerman ES, Heibeck TH, Gill A et al (2014) Production of site-specific antibody-drug conjugates using optimized non-natural amino acids in a cell-free expression system. Bioconj Chem 25:351-361 